\title{
A fluorescence spectroscopic method for rapid detection of bacterial endospores: Proof of concept
}

Nancy Awasti and Sanjeev Anand*

\section{Graphical Abstract}

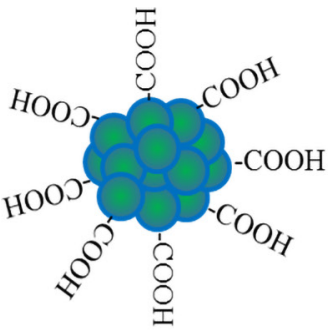

Functionalized PFO dots

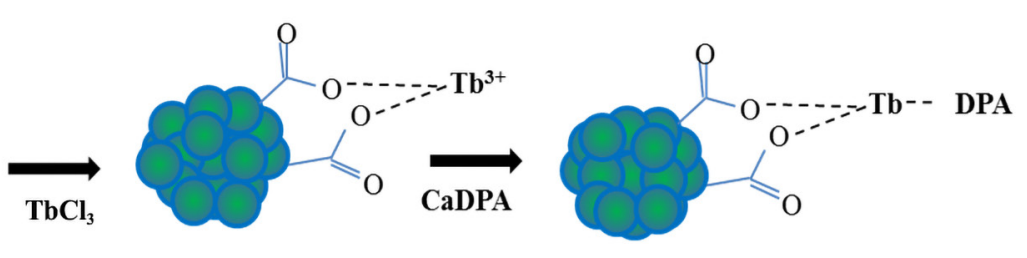

Terbium chelated functionalized PFO dots
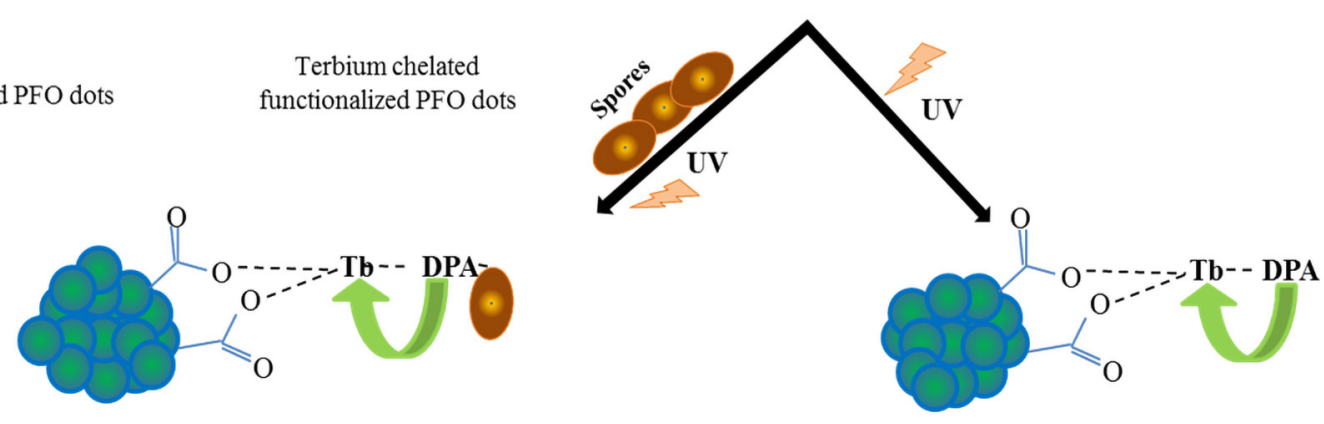

Quantification of dipicolinic acid (DPA) on spores

\section{Summary}

Bacterial endospores are resistant microbial structures that survive adverse conditions and cause product spoilage. Rapid detection of bacterial spores in dairy and food products and processing environments can thus help reduce spoilage and shelf-life issues in the final product. This study focused on evaluating a rapid, sensitive, and accurate method to quantify Bacillus spores in milk. The concentration of calcium dipicolinic acid (CaDPA) in Bacillus spores was investigated using a ratiometric fluorescence technique. CaDPA was quantified by recording the intensity (excitation-emission wavelengths 275-544 nm) of CaDPA attached to chelated semiconducting fluorescent polyfluorene (PFO). The mean CaDPA content of spiked spores detected in raw milk samples was approximately $2.5,3.8$, and $5.0 \mathrm{nM}$ for spiked levels of $5.21,6.39$, and $9.47 \log \mathrm{cfu} / \mathrm{mL}$, respectively.

\section{Highlights}

- Bacterial spores in milk and milk products can be detected by a rapid optical method.

- Chelated semiconducting polymer dots quantify CaDPA on spores.

- Proteins and ions in the sample may interfere with luminescence.

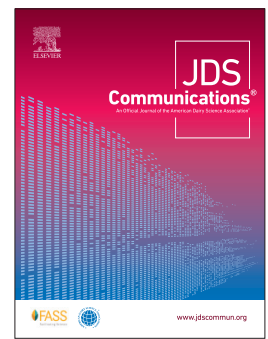




\title{
A fluorescence spectroscopic method for rapid detection of bacterial endospores: Proof of concept
}

\author{
Nancy Awasti and Sanjeev Anand*
}

\begin{abstract}
Current spore detection methods rely on culture techniques, with limitations of time, efficiency, and sensitivity. The bacterial spore coat contains calcium dipicolinic acid (CaDPA) as a major constituent, which could serve as a biomarker for bacterial endospores. We report proof of concept for a rapid and sensitive technique for the detection of bacterial endospores by using ratiometric fluorescencebased sensors. This method is based on the detection of CaDPA, which enhances the luminescence of lanthanide ions when complexed with a semiconducting polymer. A CaDPA standard curve was generated at an excitation-emission wavelength $(\lambda)$ of $\lambda_{275}-\lambda_{544}$ by using a spectrophotometer. The intensity was recorded after chelating semiconducting fluorescent polyfluorene (PFO) dots with terbium (lanthanide) ions, sensitized by different volumes of CaDPA $(0.1 \mu M)$. The resultant standard curve showed a linear relationship $\left(\mathrm{R}^{2}=0.98\right)$ in the experimental concentration range of 2.5 to $25 \mathrm{n} M$ CaDPA, with corresponding intensity (arbitrary units) of 545 to 2,130. Endospores of the aerobic sporeformer Bacillus licheniformis ATCC 14580 were produced at $37^{\circ} \mathrm{C}$ for $15 \mathrm{~d}$ on brain heart infusion agar plates. The efficiency of sporulation was evaluated by spore staining and plating techniques. Total CaDPA content on spores was estimated after suspending decreasing concentrations of spores (logs 9.0 through $1.0 \mathrm{cfu} / \mathrm{mL}$, at $1-\log$ intervals) in HPLC-grade water (to serve as control) and skim milk samples. In HPLC-grade water, for higher spiking levels such as (mean $\pm \mathrm{SD}$ ) 9.2 $\pm 0.03,8.4 \pm 0.05,7.1 \pm 0.13$, and $6.3 \pm 0.02 \mathrm{logs}$, the corresponding mean CaDPA from the standard curve were 9.4, 7.2, 6.2, and 5.3 nM, respectively. For lower spiking levels of $4.2 \pm 0.05,3.1 \pm 0.04,2.0 \pm 0.11$, and $1.36 \pm 0.09 \operatorname{logs}$, we observed mean CaDPA contents of 3.8, 3.3, 2.2, and 1.3 $\mathrm{n} M$, respectively. For raw skim milk spiked with B. licheniformis ATCC 14580 spores, the mean CaDPA content on spores was approximately 2.5, 3.8, and 5.0 $\mathrm{n} M$ for spiking levels of 5.21, 6.39, and $9.47 \log \mathrm{cfu} / \mathrm{mL}$, respectively. Trials were conducted in replicates of 3 and means were compared. Trials conducted using HPLC-grade water showed a linear relationship for the CaDPA content of endospores and for endospore counts with the standard CaDPA concentration curve. For skim milk-spiked samples, we observed reduced fluorescence detection, which was 5 times lower than that of spiked samples in HPLC-grade water. The reduced fluorescence in skim milk could be due to the turbidity of the solution or to interference from proteins, amino acids, and other ions in milk. This study thus provides proof of concept for a potential application of this technique to rapidly detect bacterial endospores in the dairy and food industry. Further work is required to remove the interference of ionic components in milk to improve detection limits in milk and other dairy product matrices such as cheese, whey proteins, and reconstituted powders.
\end{abstract}

B acterial endospores are very resistant microbial structures that survive adverse conditions and can germinate when conditions are favorable. Rapid detection of bacterial spores in dairy and food processing environments, water, and dairy and food matrices can help reduce spoilage and prevent shelf-life issues in the final product. The aerobic spore-forming Bacillus species is a major contaminant in the food and dairy industry (Seale et al., 2015). Bacillus licheniformis, being the predominant species, is frequently isolated from raw milk and responsible for significant shelf-life issues of milk and milk products (Sharma and Anand, 2002; Awasti et al., 2019). Detection and enumeration of bacterial endospore concentrations are time-consuming tasks. Therefore, timely detection of sporeformers before processing of milk is vital to identify the source of contamination and develop strategies to reduce or control Bacillus build-up. Several methods have been used previously, including plating techniques and molecular and optical methods. The most frequently used methods to quantify spores are microscopy and plate culture (counting) methods, which are slow and tedious, and may take up to $72 \mathrm{~h}$ for the results to be available. Molecular methods usually require costly reagents and require sample processing time before analysis. Thus, there remains a need for a simple and cost-effective method that can be used for the rapid identification of aerobic Bacillus spores in food and dairy matrices. For the past 2 decades, optical methods for the detection and enumeration of spores based on dipicolinic acid (DPA) have garnered attention. Some previous methods evaluated DPA as a spore marker (Bell et al., 2005) with a detection limit of 2 nM DPA. A study by Hindle and Hall (1999) quantified spores by monitoring spore germination and measured exuded DPA with minimum detection limit of $10^{4}$ Bacillus subtilis spores $/ \mathrm{mL}$.

To develop a rapid, sensitive, and accurate method to quantify spores in the food matrix, we investigated the concentration of calcium dipicolinic acid (CaDPA) in Bacillus spores using a ratiometric fluorescence technique. The strain Bacillus licheniformis ATCC 14580 was selected for the current study because of its prevalence and tendency for sporulation (Awasti et al., 2019). As a follow-up to a previous study (Li et al., 2013), we applied an optical method to detect bacterial spores based on detection of $\mathrm{CaDPA}$, an essential biomarker and major component of bacterial spores. An enhanced emission peak with bright luminescence was observed at $544 \mathrm{~nm}$ upon binding of DPA with lanthanide ion (terbium, $\left.\mathrm{Tb}^{3+}\right)$, whereas the emission peak at $439 \mathrm{~nm}$ remained stable 

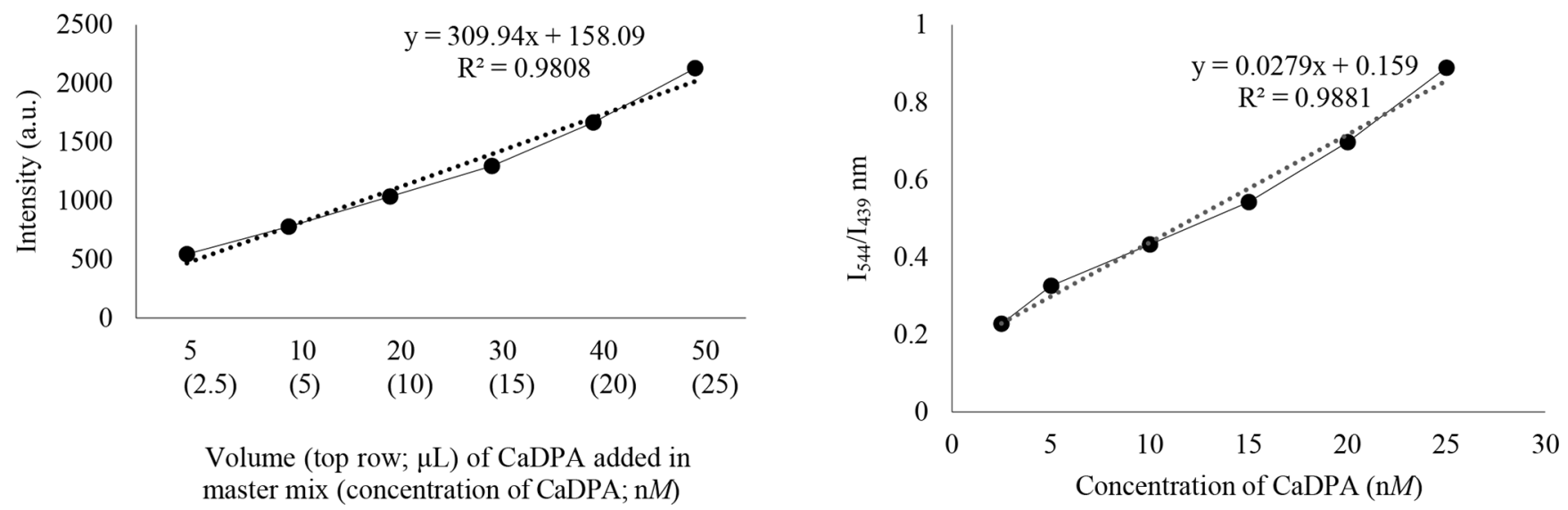

Figure 1. (A) Plot of change in luminescence intensity ( $544 \mathrm{~nm}$; arbitrary units, a.u.) of the polyfluorene dot (Pdot) sensor with increasing concentrations of calcium dipicolinic acid (CaDPA). (B) Ratiometric calibration (intensity at 544 and $439 \mathrm{~nm} ; \mathrm{I}_{544} / \mathrm{I}_{439}$ ) of the Pdot sensor as a function of CaDPA concentration.

without addition of DPA and thus served as an internal reference (Awasti and Anand, 2018). This technique had improved detection and sensitivity when spores were spiked in HPLC-grade water, but reduced fluorescence when skim milk samples were spiked with spores. This study thus provides proof of concept for the application of this technique to rapidly detect Bacillus spores in the dairy and food industry, although further work is needed to reduce ionic interference in complex matrices.

Our strategy to quantify the total number of spores in ion-free water and milk was based on the detection of CaDPA content by using ratiometric fluorescence techniques, which were previously described by Li et al. (2013). Functionalized polyfluorene (PFO) dots were prepared using the fluorescent semiconducting polymer PFO and functional polymer poly(styrene-co-maleic anhydride) in tetrahydrofuran (THF). The detection of CaDPA using the fluorescence spectroscopic technique depends on the common absorption peak ( $\square 275 \mathrm{~nm}$ ) of CaDPA and the semiconducting polymer PFO. The terbium-dipicolinic acid (Tb-DPA) complex and PFO dots can be excited simultaneously at a wavelength of $275 \mathrm{~nm}$ without affecting the luminescence of each other. In the current study, a long-pass filter was placed in front of the detector to remove any interference from excitation at $275 \mathrm{~nm}$, as reported previously (Li et al., 2013). Lanthanide ions $\left(\mathrm{Ln}^{3+}\right)$ have a high affinity for CaDPA, and thus their binding can enable a very sensitive assay with bright luminescence. Most lanthanide-based sensors only use the change in fluorescence intensity to estimate the concentration of CaDPA, whereas the ratiometric fluorescent detection method evaluated in this study can measure the relative changes in fluorescence intensities at 2 wavelengths. The exact concentration of the analyte (in this case, CaDPA) can thus be quantitatively determined using the self-calibration curve of the ratiometric sensor. Ratiometric sensors have excellent sensitivity and selectivity, with a detection limit of $0.2 \mathrm{n} M$, reportedly the best among different CaDPA sensors.

Functionalized Pdots were prepared using the nanoprecipitation method described in a previous study (Li et al., 2013). The CaDPA solution was prepared by neutralizing reaction and stored at $5^{\circ} \mathrm{C}$ for $48 \mathrm{~h}$, followed by filtration and evaporation. For CaDPA sensor detection, terbium chloride $\left.(0.1 \mathrm{mM} \mathrm{TbCl})_{3}\right)$ was added to an aqueous solution of prepared functionalized PFO dots to produce a reaction mixture with a Pdot concentration of about $80 \mathrm{p} M$ and a terbium concentration of $1 \mu M$. The solution was agitated for $5 \mathrm{~min}$ and then luminescence sensing of the terbium-chelated PFO dots was performed by adding different volumes of CaDPA $(0.1 \mu M)$ to the terbium-chelated Pdot solutions. Fluorescence spectra were measured using a Synergy 2 fluorescence spectrometer (BioTek Instruments Inc.). First, a standard curve was prepared by using different concentrations of CaDPA at luminescence intensity of $544 \mathrm{~nm}$ (Figure 1A), and then, ratios of intensities (I) were plotted at 544 and $439 \mathrm{~nm}\left(\mathrm{I}_{544} / \mathrm{I}_{439}\right)$ to generate a CaDPA calibration curve (Figure 1B). In the next step, the protocol was validated by spiking spores in HPLC-grade water. Following validation, a similar protocol was followed to determine and quantify the spores present in raw skim milk samples.

This study used Bacillus licheniformis ATCC 14580, purchased from the American Type Culture Collection (ATCC). The strain was propagated at $37^{\circ} \mathrm{C}$ in brain heart infusion agar (BHI; BD Difco), and endospores were prepared using the method described by Awasti and Anand (2018). To monitor the level of sporulation, spore staining was performed occasionally throughout the $15-\mathrm{d}$ incubation period; after achieving $90 \%$ sporulation, spores were collected using centrifugation $(34,000 \times g, 15 \mathrm{~min})$ followed by washing with sterile distilled water, as described by Wang et al. (2009) and Awasti (2019). Total CaDPA counts of spores spiked in HPLC-grade water and in skim raw milk samples were estimated by spiking decreasing concentrations of spores $(\operatorname{logs} 9.0$ through $1.0 \mathrm{cfu} / \mathrm{mL}$, at 1-log intervals) separately in HPLC-grade water and raw skim milk. The spiked samples were analyzed using the Synergy 2 fluorescence spectrometer (BioTek Instruments Inc.). Samples were excited at a wavelength $(\lambda)$ in the UV range (i.e., $\lambda_{275}$ ), and fluorescence was read at 2 intensities: $\mathrm{I}_{544}$ and $\mathrm{I}_{439}$. The ratios of the 2 intensities were separately plotted for both sample 
A

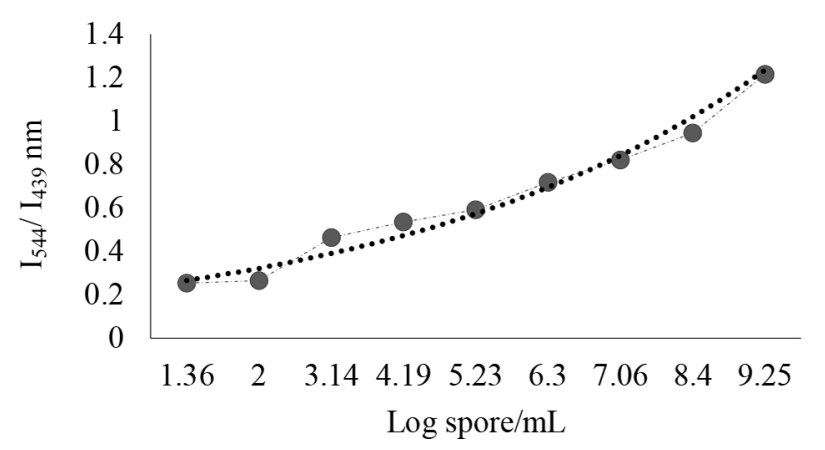

B

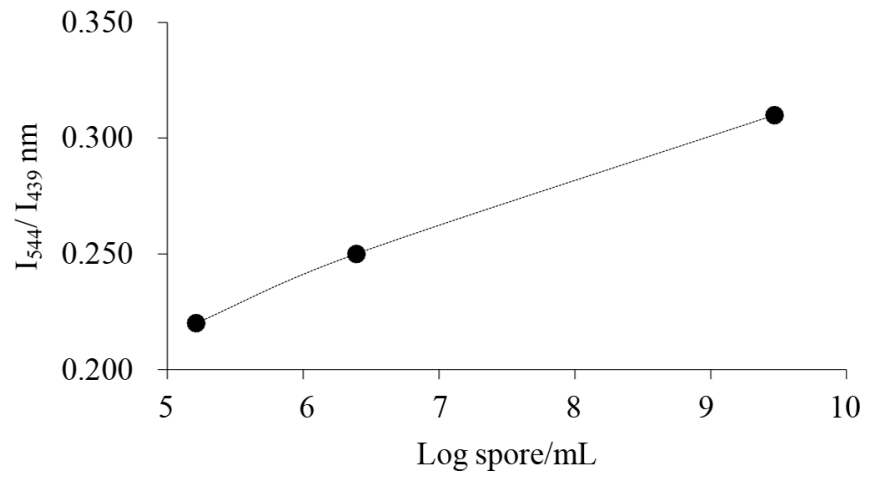

Figure 2. Ratiometric calibration plot (intensity at 544 and $439 \mathrm{~nm} ; \mathrm{I}_{544} / \mathrm{I}_{439}$ ) of spores spiked in (A) HPLC-grade water and (B) skim milk samples using the polyfluorene dot (Pdot) sensor as a function of calcium dipicolinic acid (CaDPA) concentration.

types (Figure 1A and 1B). The mean CaDPA concentrations in spore-spiked HPLC-grade water and raw skim milk samples were quantified using the CaDPA calibration curve (Figure 1A and 2B).

According to Li et al. (2013), the sensitivity of the CaDPA sensor was evaluated and their results showed a significant luminescence response of the sensor when CaDPA in aqueous solution was excited at $275 \mathrm{~nm}$. The emission spectra of PFO dots and CaDPA do not interfere with each other, and their major emission peaks were at 439 and $544 \mathrm{~nm}$, respectively. Our results validated those of the previous study by plotting the luminescence intensity with increasing CaDPA concentration. Our results agreed with those of $\mathrm{Li}$ et al. (2013) in terms of excitation and emission of CaDPA sensors; CaDPA was detected and plotted at excitation-emission $\left(\lambda_{275}-\lambda_{544}\right)$ wavelengths using the Synergy 2 fluorescence spectrophotometer (Figure 1A). A linear relationship $\left(\mathrm{R}^{2}=0.98\right)$ was observed for our experimental CaDPA concentration range of 2.5 to $25 \mathrm{n} M$ with corresponding intensity (arbitrary units, a.u.) of 545 to 2,130.

Figure 1B explains the ratiometric calibration plot $\left(\mathrm{I}_{544} / \mathrm{I}_{439}\right)$ of the Pdot sensor as a function of CaDPA concentration. The ratios of 2 emission intensities were plotted where the highest emission peaks of $\mathrm{Tb}^{3+}$ were excited after chelating it between DPA and functionalized PFO dots. In terms of sensor sensitivity, our results agree with the previous report, and the limit of detection observed was approximately $0.2 \mathrm{n} M$. The results shown in Figure 1B allowed us to create a link between CaDPA concentration and ratiometric intensity, which was further used to calibrate the total CaDPA content of spores spiked in HPLC-grade water and raw skim milk samples. After exciting the reaction mixture of spores spiked in HPLC-grade water at $275 \mathrm{~nm}$, luminescence was observed at 2 intensities: $\mathrm{I}_{544}$ and $\mathrm{I}_{439}$. The ratios of these 2 intensities were plotted against spiked $\log$ spore counts $(\log \mathrm{cfu} / \mathrm{mL})$, as shown in Figure 1A. This graph was compared with the CaDPA calibration curve (Figure 1B) to quantify the total concentration of CaDPA on spores. For higher spiking levels such as $9.2 \pm 0.03,8.4 \pm 0.05$, $7.1 \pm 0.13$, and $6.3 \pm 0.02 \operatorname{logs}$, the corresponding mean CaDPA values determined from the standard curve were 9.4, 7.2, 6.2, and $5.3 \mathrm{n} M$, respectively. For the lower levels of $4.2 \pm 0.05,3.1 \pm 0.04$, $2.0 \pm 0.11$, and $1.36 \pm 0.09 \mathrm{logs}$, we determined mean CaDPA concentrations of $3.8,3.3,2.2$, and $1.3 \mathrm{n} M$, respectively. The mean
CaDPA concentration on spores ranged from approximately 30 to $3 \mathrm{n} M$. Trials conducted using HPLC-grade water indicated a linear relationship of CaDPA content of endospores with the endospore counts and the standard curve of CaDPA concentration.

For raw skim milk spiked with B. licheniformis ATCC 14580 spores, the mean CaDPA concentrations detected on spores were approximately $2.5,3.8$, and $5.0 \mathrm{n} M$ for spiking levels of 5.21, 6.39, and $9.47 \log \mathrm{cfu} / \mathrm{mL}$, respectively (Figure $2 \mathrm{~B}$ ). Under our test conditions, we could not detect intensities below the spiking level of $5.21 \log \mathrm{cfu} / \mathrm{mL}$. The fluorescence detection was approximately 5 times lower for spiked samples in skim milk compared with those in HPLC-grade water. The reduced fluorescence in raw milk may be due to the turbidity of the solution or interference from proteins, amino acids, and other ions present in milk. To improve the ability of the sensor, additional strategies are needed to remove interfering components such as proteins and other ions. This study supports the potential application of this technique to rapidly detect bacterial endospores in the dairy and food industry. Further refinements are needed to remove the interference of ionic components in milk and improve the efficiency of the sensor to rapidly detect spores.

\section{References}

Awasti, N. 2019. Influence of sporulation and germination behavior of Bacillus licheniformis on microbial quality of skim milk powder. $\mathrm{PhD}$ thesis. South Dakota State University. https://openprairie.sdstate.edu/etd/3358/.

Awasti, N., and S. K. Anand. 2018. Ratiometric fluorescence spectroscopy-A novel technique for rapid detection of bacterial endospores. J. Dairy Sci. 101(Suppl. 2):9. (Abstr.)

Awasti, N., S. K. Anand, and G. Djira. 2019. The sporulating behavior of Bacillus licheniformis strains influences their population dynamics during raw milk holding. J. Dairy Sci. 102:6001-6012. https://doi.org/10.3168/jds .2018-15613.

Bell, S. E. J., J. N. Mackle, and N. M. S. Sirimuthu. 2005. Quantitative surfaceenhanced Raman spectroscopy of dipicolinic acid-towards rapid anthrax endospore detection. Analyst 130:545-549. https://doi.org/10.1039/ B415290E.

Hindle, A. A., and A. H. E. Hall. 1999. Dipicolinic acid (DPA) assay revisited and appraised for spore detection. Analyst 124:1599-1604. https://doi.org/ 10.1039/a906846e.

Li, Q., K. Sun, K. Chang, J. Yu, D. T. Chiu, C. Wu, and W. Qin. 2013. Ratiometric luminescent detection of bacterial spores with terbium chelated semiconducting polymer dots. Anal. Chem. 85:9087-9091. https://doi.org/ 10.1021/ac4016616. 
Seale, B., P. Bremer, S. Flint, J. Brooks, and J. Palmer. 2015. Overview of the problems resulting from biofilm contamination in the dairy industry. Pages 49-64 in Biofilm in the Dairy Industry. K. H. The, S. Flint, J. Brooks, and G. Knight, ed. Wiley. https://doi.org/10.1002/9781118876282.ch4.

Sharma, M., and S. Anand. 2002. Characterization of constitutive microflora of biofilms in dairy processing lines. Food Microbiol. 19:627-636. https://doi .org/10.1006/fmic.2002.0472.

Wang, B. S., B. S. Li, Q. X. Zeng, J. Huang, Z. Ruan, Z. W. Zhu, and L. Li. 2009. Inactivation kinetics of reduction of Bacillus coagulans spore by the combination of high pressure and moderate heat. J. Food Process Eng. 32:692-708. https://doi.org/10.1111/j.1745-4530.2007.00239.x.

\section{Notes}

This work was funded by the Midwest Dairy Association (St. Paul, MN)

The authors acknowledge the Functional Genomics Core facility for use of Synergy 2 fluorescence spectrophotometer, and the Agricultural Experimentation Station, South Dakota State University, in conducting this study.

The authors have not stated any conflicts of interest. 